\title{
Quality and safety of handover in intensive care
}

\author{
EM Siriwardena $^{1 *}$, AD Mudalige ${ }^{2}$ \\ Senior Registrar in Anaesthesiology and Intensive Care ${ }^{I^{*}}$, Senior Registrar in Critical Care ${ }^{2}$ \\ National Hospital of Sri Lanka. Colombo, Sri Lanka.
}

\begin{abstract}
Introduction
Handover is an inevitable and essential aspect of caring for critically ill patients and information handed over should be accurate, succinct, and sufficient to allow the seamless continuation of care between teams.

Objectives

To assess staff involvement, time taken for the handover, key components addressed, availability of a standard format and factors causing disturbances to an effective handover were the key components studied to determine the quality and safety.

Methodology

Study population were doctors and nursing offices working in the general and specialised ICUs of the National Hospital of Sri Lanka, De Soysa maternity hospital and the Castle street hospital for women. Study period was July to September 2013. Sample size was 120. Data was collected using a self-administered questionnaire based on the guidelines on clinical handover by the Australian Medical Association. Data was analysed as simple percentages and review of absolute numbers using bar and pie charts for each ICU.

Results

The doctor's presence in the nurse's handover and nurse's presence in doctor's handover was less than $70 \%$ in all the ICUs. There was no fixed time for handover in $92 \%$ of ICU shifts and nearly $90 \%$ of times it happened after the conclusion of the shift and the average time taken was 30mins. Compliance with the recommended content of handover was more than $60 \%$ in almost all the ICUs. There was no structured format for handover in any of the ICUs. Telephone calls were the most common distracting factor identified. Conclusion / Recommendations

Handover can be made more effective and safe with implementation of a printed handover sheet for use as well as an overlapping shift pattern in duty rosters, dedicated time and a place for handover.
\end{abstract}

Keywords: Handover; intensive care; quality; safety; shift work

\section{Introduction}

Handover is an inevitable and essential aspect of caring for critically ill patients whilst working according to a shift system.

Clinical handover between shifts is necessary to ensure that information about patient care is correctly transmitted between incoming and outgoing medical teams. The information
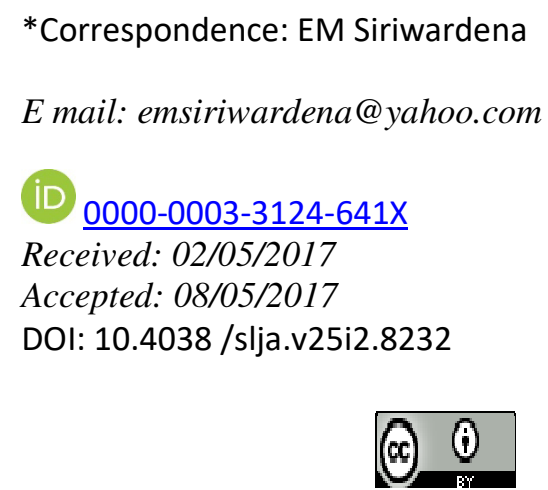

handed over should be accurate, succinct, and sufficient to allow the seamless continuation of care between teams.

Breakdown in communication is one of the leading causes of patient harm and therefore handover is a key component of safe patient care in the critical care setting. ${ }^{1}$ The handover should occur at a designated time in a designated area with clear leadership and without avoidable interruption. Information must be up to date. Ideally a standardized format of presentation should be used to ensure key information is not omitted. $^{2}$

Inadequate handover carries risks for patients, individual clinicians and the organizations within which they work. ${ }^{1,3,4}$ Current handover practices are often not standardized and are highly variable. ${ }^{2}$ 


\section{General objective}

To assess the overall quality, nature and effectiveness of the handover process in the intensive care units.

\section{Specific objectives were to assess}

1. the degree of staff involvement in the handover process.

2. the time taken for the handover and its impact on the shift system.

3. the key components addressed during the handover process.

4. the availability of a standard format to be used for handover.

5. the influence of factors creating a hindrance to an effective handover.

\section{Methodology}

The study was carried out among Doctors (medical officers and postgraduate trainees) and nursing officers working in the general surgical, trauma, cardiothoracic, neurosurgical and medical ICUs of the National Hospital of Sri Lanka and in the obstetric ICUs of the De Soysa maternity hospital and the Castle street hospital for women during the period from $1^{\text {st }}$ July 2013 to $30^{\text {th }}$ September 2013. The sample size was 120 .

A self-administered questionnaire was developed with the help of the guidelines on clinical handover, proposed by the Australian Medical Association in 2006. It was developed in English. Pretesting the questionnaire was done amongst ten participants to ensure the validity, reliability and clarity of the questionnaire and necessary changes were made. Ethical clearance was obtained from the ethical review committees of each hospital and of the Post Graduate Institute of Medicine, University of Colombo.

During the period of study, questionnaires were handed over to each and every doctor and nurse (who consented to participate in the audit) in a particular shift. Each of the three major shifts were included once in the study, in a given ICU during the period of study. Informed written consent was obtained after explaining the study objectives. The participants were given required instructions, time and privacy to respond to the questions. Strict anonymity was maintained and all data were kept confidential.
Data was analysed as simple percentages and review of absolute numbers using bar and pie charts. Each component of the questionnaire was analysed separately.

\section{Results}

Demographic data

$42 \%$ of the study population were doctors and $58 \%$ were nurses.

The highest percentage of doctors and nurses in the sample were from general surgical, neurosurgical, cardiothoracic and obstetric ICUs.

\section{Categories of staff present for the handover}

Ideally members of all the teams involved in the care of patients should be present for the hand over. Eg:- ICU doctors, doctors of relevant medical/surgical unit, nurses, paramedical staff etc.

A key issue noticed in our setup is that there is no single handover involving all staff categories during any of the shifts concerned. The doctors' and nurses' shifts operate at different hours and handover occur separately among the doctors and the nurses.

Overall the nurse participation in doctor's handover was better in all the ICUs except for the medical ICU when compared to the medical officer participation in the nurse's handover.

Table 1: Staff presence during handover

\begin{tabular}{|c|c|c|c|c|c|c|c|c|}
\hline $\begin{array}{l}\text { Doctis's } \\
\text { handber }\end{array}$ & Totat & $\frac{G \text { en }}{\underline{C U}}$ & $\frac{\text { Tram }}{\mathrm{a} \text { a }}$ & $\frac{\text { Cad }}{\underline{\underline{W}}}$ & $\frac{\text { Navo }}{\underline{\underline{\mathbf{c} U}}}$ & $\frac{\mathrm{Med}}{\underline{\underline{\mathbf{W}}}}$ & $\begin{array}{l}\frac{\underline{D M H}}{\underline{\mathrm{dbs}_{\mathrm{S}}}} \\
\underline{\underline{\underline{U}}}\end{array}$ & $\begin{array}{l}\frac{C \mathrm{CW}}{\mathrm{HObS}} \\
\underline{\underline{\mathrm{DU}}}\end{array}$ \\
\hline 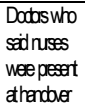 & $47 \%$ & $70 \%$ & $40 \%$ & ఝ\% & $50 \%$ & $\%$ & $\varpi \%$ & $25 \%$ \\
\hline $\begin{array}{l}\text { Dodasuhto } \\
\text { sadruses } \\
\text { weend } \\
\text { pesertat } \\
\text { hendber }\end{array}$ & $53 \%$ & $30 \%$ & $\bigoplus \%$ & $30 \%$ & $50 \%$ & $100 \%$ & $40 \%$ & $\pi 5 \%$ \\
\hline $\begin{array}{l}\text { Nusets } \\
\text { handber }\end{array}$ & Tota & $\frac{G \text { en }}{\underline{C U}}$ & $\frac{\text { Tram }}{\text { ald }}$ & $\frac{\text { Cad }}{\underline{\underline{w}}}$ & $\frac{\text { Navo }}{\underline{C U}}$ & $\frac{\text { Mod }}{\underline{\mathbf{C W}}}$ & $\begin{array}{l}\frac{D M H}{O D S} \\
\underline{\underline{D U}}\end{array}$ & $\begin{array}{l}\frac{\mathrm{CSW}}{\mathrm{HOO_{BS }}} \\
\underline{\mathrm{CU}}\end{array}$ \\
\hline $\begin{array}{l}\text { Nuses vho } \\
\text { sad dodas } \\
\text { wee pereft } \\
\text { thencher }\end{array}$ & $3 \%$ & 0 & $\%$ & $\%$ & $20 \%$ & $\%$ & $\%$ & $0 \%$ \\
\hline 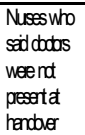 & $9 \%$ & $100 \%$ & $100 \%$ & $100 \%$ & $80 \%$ & $100 \%$ & $100 \%$ & $100 \%$ \\
\hline
\end{tabular}


When the handover should take place?

According to the AMA guidelines handover should be at a fixed time and of a sufficient time (30 minutes or more) within the shift concerned. Shifts of all staff must be coordinated to allow them to attend the handover meeting within working hours and the time of the handover meeting should be known to all.

$>$ Presence of a fixed time for handover

There was no fixed time for the doctor's handover in any of the ICUs. In the general surgical ICU, cardiothoracic ICU and the obstetric ICU at CSHW the nurse's handover occurred at a fixed time $50 \%$ or more of the times.

$>$ Handover occurring within / outside the shift Majority of the doctor's handover occurred outside the shift but the nurse's handover in the trauma ICU, medical ICU and the obstetric ICU at CSHW occurred $50 \%$ or more of the times within the respective shift.

$>$ Time taken for the handover

Except for the doctor's handover in the MICU all the other handovers were completed in less than 30 minutes.

$>$ Where should the handover take place?

It should be a designated, large enough comfortable place that can accommodate the number of people involved, situated close to the most used working area and be devoid of distractions. It should ideally have access to lab results, $x$-ray viewing, telephone etc.

All handovers in our ICUs by the doctors as well as the nurses occurred at the patient's bedside.

$>$ What should be handed over?

In the ideal setup the general introduction and diagnosis, important events that occurred in the past shift, current situation and plans for the next shift should all be handed over.

A significant proportion of doctors (69\%) failed to mention the patient's name during the handover. All other factors were mentioned quite satisfactorily by the doctors as well as nurses.

Handing over information regarding discussions with the family was markedly lacking in the handover process among the doctors as well as nurses with only $31 \%$ of the doctors and $38 \%$ of the nurses paying attention to the fact.
Figure 1: Information handed over on general introduction and diagnosis

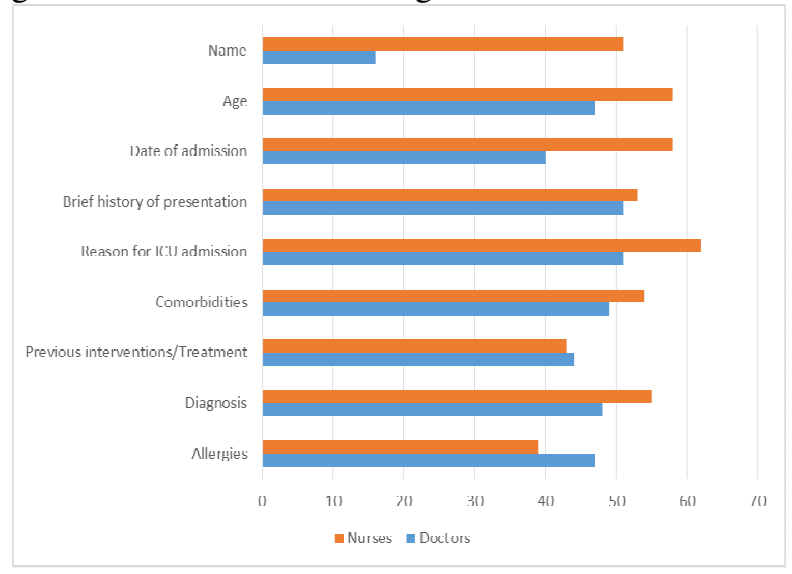

Figure 2: Information handed over on important events that occurred in the past shift

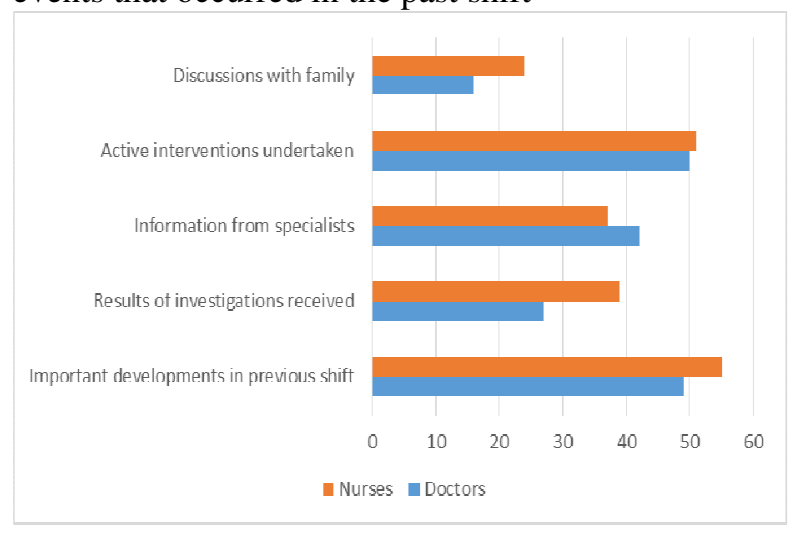

Information about the current situation $100 \%$ of the doctors and $90 \%$ of the nurses have mentioned the present problems involved in patient's care during the handover.

Figure 3: Information handed over about plans for the next shift

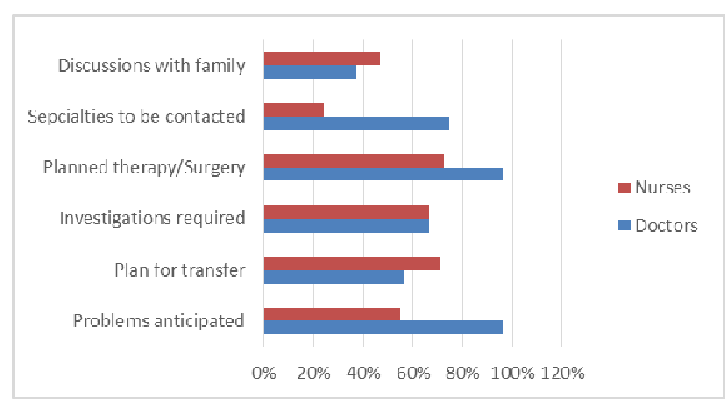


Again, plans for relevant discussions with the family during the next shift was a factor that was paid least attention to during the handover process by the doctors as well as the nurses.

$>$ Format of handover

This should ideally be written or computer based.

In all the ICUs under study all handovers among the doctors and nurses were carried out verbally with no involvement of a written document.

\section{Distractions during the handover}

The handover meeting should ideally be free of disturbances from bleeps, pagers, telephones, relatives, nurses and other doctors unless for a major emergency.

Table 2: Common factors distracting the handover process

\begin{tabular}{|l|r|r|}
\hline Distractions & \multicolumn{2}{|c|}{ Total } \\
\hline & \multicolumn{1}{|c|}{ Doctors } & \multicolumn{1}{c|}{ Nurses } \\
\hline Other Medical Officers & $29 \%$ & $30 \%$ \\
\hline Nurses & $43 \%$ & $42 \%$ \\
\hline Other Staff & $22 \%$ & $28 \%$ \\
\hline The Patient & $14 \%$ & $19 \%$ \\
\hline Other Patients & $31 \%$ & $25 \%$ \\
\hline Relations & $4 \%$ & $17 \%$ \\
\hline Phone Calls & $90 \%$ & $81 \%$ \\
\hline Bleeps & $0 \%$ & $3 \%$ \\
\hline $\begin{array}{l}\text { External Noise } \\
\text { (TV/Audio/Alarms etc) }\end{array}$ & $29 \%$ & $28 \%$ \\
\hline
\end{tabular}

The most common factor causing distractions during handovers in all of the ICUs were telephone calls.

\section{Discussion}

This study focuses on several key components of an effective clinical handover in an ICU setting that ensures safe and effective continuity of care.

One main drawback for an effective and safe handover noted in all of the ICUs concerned, was the absence of a single handover meeting conducted involving all of the team members. This falls short of the recommended standards stated by the Australian Medical Association ${ }^{3}$ as well as the National Patient Safety Agency ${ }^{1}$ of the United Kingdom. The doctor's and the nurse's handover occur separately in all of our ICUs. The participation of nurses in the doctors' handover (47\%) was significantly better than the doctor's participation in the nurses' handover $(3 \%)$.
All though the recommended standard for a handover is to have a fixed time within the shift of a reasonable duration of thirty minutes or more, this standard was not met in the handovers that occurred in our setup. Overall $92 \%$ of the doctors and $62 \%$ of the nurses stated that there is no fixed time for the handover. All doctors $(100 \%)$ in the general surgical, medical, trauma, cardiothoracic, neurosurgical and DMH obstetric ICUs stated that there is no fixed time for handover. Only $50 \%$ of the doctors working in the CSHW obstetric ICU seem to keep a fixed time for the handover. A great majority of the doctors' handovers occurred after the shift. Only in the cardiothoracic $(71 \%)$ and CSHW obstetric ICU $(70 \%)$ that a significant proportion of the nurses handed over within the shift. Majority of the doctors and nurses in all of the ICUs took less than thirty minutes for the handover process, falling short of the standard.

There is no designated, well equipped, spacious enough place in any of the ICUs to hold a handover meeting and it occurred exclusively at the patient's bedside.

Considering the information that was handed over, many aspects such as general information, diagnosis, important events in the past shift, details of the current situation and plans for the next shift were satisfactorily handed over by both doctors and nurses. Both groups failed to emphasise on discussions with the family of the patient and a significant proportion of doctors (69\%) failed to mention the patient's name during the handover.

The absence of a written or IT based format to use for the handover is another major drawback that was noted in our system.

The most common factor distracting proper handover was phone calls. However, nurses, medical officers and patients also caused disturbances in most of the ICUs.

\section{Conclusion}

The following aspects of the clinical handover did not meet the recommended standards in any of the ICUs under study and needs improvement:

o Not holding a common handover meeting for each shift. 
- Not having overlapping working hours to hold a handover meeting of thirty minutes or more at a fixed time within working hours.

- Not having a designated place that is free of distractions to hold the handover meeting.

- Not having a written or IT based format to use for the handover.

However, handing over of important information related to patient care was satisfactory and met the recommended standards in all of the ICUs amidst the above constraints. Involvement of family members and related information handover needs improvement among doctors as well as nurses.

\section{Recommendations}

$>$ Implementation of a duty roster that enables working hours to overlap between the shifts.

$>$ Allocating a dedicated time at the beginning of each shift for team members to meet, share information and clarify responsibility for ongoing patient care.

$>$ Implementation of a printed handover sheet to be used for each shift change.

\section{References}

1. Safe handover: safe patients - guidance on clinical handover for clinicians and managers. Hospital at Night Risk Assessment Guide, London. National Patient Safety Agency, 2004.

2. Lyons MN, Standley TDA, Gupta AK. Quality improvement of doctors' shift-change handover in neuro-critical care. Qual Saf Health Care 2010;19:1-7.

https://doi.org/10.1136/qshc. 2008.028977

3. Safe handover: safe patients. Guidance on clinical handover for clinicians and managers, by the Australian Medical Association.

4. Safe handover: Guidance from the working time directive working party. The Royal College of Surgeons of England, March 2007. 